Recepción: 20 / 04 / 2017

Aceptación: 20 / 05 / 2017

Publicación: 15 / 07 / 2017

Ciencias de la Salud

Artículo de Investigación

\title{
Rehabilitación protésica fija con puente metal porcelana
}

\author{
Fixed prosthetic rehabilitation with metal porcelain bridge
}

Fixa restauração protética com ponte de porcelana de metal

Miguel A. Rodriguez-Llaguno ${ }^{\text {I }}$ miguel.rodriguezll@ug.edu.ec

Jorge L. García-Villamar II jorge.garciav@ug.edu.ec

Ernesto R. Montece-Seixas III ernesto.monteces@ug.edu.ec

Katherine E. Lima-Tamay ${ }^{\text {IV }}$ kat12_1986@hotmail.com

Correspondencia: miguel.rodriguezll@ug.edu.ec

Diplomado en Docencia Superior; Magister en Docencia y Gerencia en Educación Superior; Diplomado en Docencia Superior; Doctor en Odontología; Universidad de Guayaquil, Guayaquil, Ecuador.

II. Magister en Diseño Curricular; Diplomado en Docencia Superior; Doctor en Odontología; Universidad de Guayaquil, Guayaquil, Ecuador.

III. Magister en Diseño Curricular; Diploma Superior en Diseño Curricular por Competencias; Diplomado en Docencia Superior; Universidad de Guayaquil, Guayaquil, Ecuador.

Iv. Odontóloga; Universidad de Guayaquil, Guayaquil, Ecuador. 
Miguel A. Rodriguez-Llaguno; Jorge L. García-Villamar; Ernesto R. Montece-Seixas; Katherine E. Lima-Tamay

\section{Resumen}

En el siglo XX cuando Edward Angle estableció una relación basada en la relación de las cúspides entre los primeros molares superiores e inferiores, la cual ha sido tomada como patrón de referencia así la de Clase I (Neutroclusión), cuando la cúspide mesio-vestibular del primer molar superior ocluye en el surco vestibular del primer molar inferior. La porcelana no reacciona con el agua destilada, pero si la mezcla se calienta a temperatura altas, sufre una reacción piroquimica; Clase II (Distoclusión), cuando la cúspide mesio- vestibular del primer molar superior ocluye por delante de la cúspide mesiovestibular del primer molar inferior; Clase III (Mesioclusión), cuando la cúspide mesio- vestibular del primer molar superior ocluye por detrás de la cúspide disto- vestibular del primer molar inferior. Las porcelanas tipo feldespáticas no fueron superadas, hasta el día de hoy, en estética por ello son bastante usadas y entre los principios de preparación que se debe tomar en cuenta también son los de preservar la estructura dentaria, retención y estabilidad solidez estructural, márgenes perfectos y preservar el periodonto.

La rehabilitación dental hace que los pacientes busquen profesionales que cuenten con alternativas de restauración por eso el material cerámico que no sufre solubilidad, desintegración, corrosión y tiene un aspecto óptico con mecánicas duradera, una estructura bifásica compuesta por vidrio ,feldespato, cuarzo y caolín necesitando de altas temperaturas para la fusión esto es una restauración dental fabricada de porcelana dando la posibilidad de imitar en forma óptica al diente natural.

Palabras claves: Rehabilitación, prótesis fija, funcionalidad, estética, sistema estomatognático. 


\section{Abstract}

In the 20th century, when Edward Angle established a relationship based on the relation between the cusps between the upper and lower first molars, which has been taken as the reference pattern, Class I (Neutroclusion), when the mesiobuccal cusp Upper molar occludes in the vestibular groove of the lower first molar. The porcelain does not react with distilled water, but if the mixture is heated to high temperatures, it undergoes a pyrochemical reaction; Class II (Dystoclusion), when the mesiobuccal cusp of the maxillary first molar occludes anterior to the mesiobuccal cusp of the lower first molar; Class III (Mesiocclusion), when the mesiobuccal cusp of the first maxillary molar occludes behind the distobuccal cusp of the lower first molar. To this day, feldspathic porcelains have not been surpassed in aesthetics, so they are quite used and among the principles of preparation that must be taken into account are also preserving the dental structure, retention and stability structural strength, perfect margins And preserve the periodontium.

The dental rehabilitation makes the patients look for professionals who have alternatives of restoration for that reason the ceramic material that does not suffer solubility, disintegration, corrosion and has an optical aspect with durable mechanics, a biphasic structure composed of glass, feldspar, quartz and kaolin needing Of high temperatures for the fusion this is a dental restoration made of porcelain giving the possibility to imitate in optical form the natural tooth.

Key words: Rehabilitation, fixed prosthesis, functionality, esthetics, stomatognathic system. 
Miguel A. Rodriguez-Llaguno; Jorge L. García-Villamar; Ernesto R. Montece-Seixas; Katherine E. Lima-Tamay

\section{Resumo}

No século XX quando Edward Ângulo estabelecido um relacionamento com base na relação das cúspides entre os primeiros molares superiores e inferiores, o que foi tomado como um padrão de referência e a Classe I (neutroclusion), quando o pico mésio-bucal de primeiro molar oclui no sulco inferior bucal primeiro molar. Porcelana não reagem com a água destilada, mas se a mistura é aquecida a alta temperatura, é submetido a uma reacção piroquimica; Classe II (distoclusão) quando a cúspide mésio vestibular do primeiro molar superior oclui em frente da altura mésio vestibular primeiro molar inferior; Classe III (mesioclusion) quando a cúspide vestibular mésio do primeiro molar superior oclui disto- atrás da cúspide vestibular do primeiro molar mandibular. Tipo de porcelanas feldspáticos não foram ultrapassadas até hoje, em estética, por conseguinte, são bastante utilizados e que os princípios da preparação a ser tomado em consideração são também preservar a estrutura do dente, de retenção e resistência estrutural a estabilidade, as margens perfeito e preservar o periodonto.

A restauração dentária torna os pacientes procuram os profissionais com opções alternativas para que o material de cerâmica que não sofre nenhuma solubilidade, desintegração, corrosão e tem uma aparência visual com mecânica durável, uma estrutura bifásica constituída por vidro, feldspato, quartzo e necessidade caulino temperatura elevada para derreter este é uma restauração dentária feita de porcelana dando a possibilidade de imitar o dente natural opticamente.

Palavras-chave: Reabilitação, prótese fixa, funcionalidade, estética, sistema estomatognático. 


\section{Introducción}

\section{Porcelana Dental}

Es un material cerámico con algunas características particulares: No sufren solubilidad, desintegración ni corrosión asegurando un aspecto óptico y propiedades mecánicas duraderas, forma una estructura bifásica al contener una fase compuesta por un vidrio y otra por cristales. Los componentes básicos son el feldespato, cuarzo y caolín. Uno de los aspectos más interesantes de las restauraciones fabricadas con porcelanas es la posibilidad de imitar el aspecto óptico del diente en forma natural especialmente respecto a su translucidez y brillo. (Villaroel, 2017)

Es un material inherentemente frágil y prácticamente nada tenaz; no posee capacidad de deformación permanente. Entendiendo a la tenacidad como una forma en que el material gana resistencia la porcelana deberá obtener otros mecanismos para mejorar su comportamiento físico, especialmente cuando las estructuras a las que dé origen funcionen en un terreno tan exigente mecánicamente como a veces es el sistema masticatorio.

Se necesita de altas temperaturas para la fusión de los componentes y permitir así la elaboración de la estructura. Posee buena resistencia a la compresión, pero baja a la tracción y más aún a la flexión lo que comúnmente expone a estos objetos a fracturas. Algunas porcelanas pueden adherirse e integrarse físicamente a su superficie consiguiendo beneficios mecánicos, ópticos y biológicos. De esta forma también se posibilita el correcto funcionamiento mecánico y la retención de algunas restauraciones.

La porcelana no reacciona con el agua destilada, pero si la mezcla se calienta a temperatura alta, sufre una reacción piroquimica. (Mendez, 2011)

\section{Según su temperatura de fusión}


Miguel A. Rodriguez-Llaguno; Jorge L. García-Villamar; Ernesto R. Montece-Seixas; Katherine E. Lima-Tamay PORCELANA DE BAJA FUSION: Esta porcelana se funde a 1600 y 19502F (871 a 10109C). Raras veces se utiliza esta porcelana para la elaboración de coronas o puentes, principalmente sirve para los contornos o agregar puntos de contacto a restauraciones de porcelana de fusión media o alta.

PORCELANA DE MEDIA FUSION: Esta porcelana se funde de 2000 a 2300eF (1090 a 12608C); nos sirve para la realización de pónticos, también nos sirve para incrustaciones, coronas y puentes.

PORCELANA DE ALTA FUSION: Esta porcelana se funde de 2400 a 25009F (1315 a 1371C); están indicadas para coronas completas y como base para las porcelanas de fusión baja o media, especialmente en la construcción de puentes de porcelanas reforzadas con metal, tiene cualidades de resistencia, color y transparencia

\section{Según la composición química}

Pueden ser:

a) Porcelanas feldespáticas: Que constan de una masa de feldespato con cuarzo y caolín dispersos. El feldespato se encarga de proporcionar translucidez a la porcelana. El cuarzo formará la fase cristalina. El caolín o arcillas, confieren plasticidad y facilitan el manejo de la cerámica cuando todavía no está cocida. A esta mezcla se añaden fundentes, opacificadores, pigmentos, etc.

Las porcelanas tipo feldespáticas no fueron superadas, hasta el día de hoy, en estética por ello son bastante usadas. 
b) Porcelanas aluminosas: Son una variación de las porcelanas tipo feldespática, porque en vez de utilizar el cuarzo se utiliza óxido de aluminio, pero no debe superar el 50\%, ya que opaca el material de restauración y se convierte en una desventaja, que es resuelta utilizándola como base que luego se recubre con una cerámica con menos del 50\% de alúmina u óxido de aluminio.

\section{Según la resistencia}

Las porcelanas se pueden clasificar en aquellas que tienen:

a) Baja resistencia: Como las porcelanas feldespáticas, con una resistencia de 100-300 MPa

b) Resistencia moderada: Representado a este grupo las porcelanas aluminosas con 300 a $700 \mathrm{MPa}$ de resistencia.

c) Alta resistencia: A este grupo pertenecen las cerámicas circoniozas con más de $700 \mathrm{MPa}$ de resistencia

\section{Según la técnica de procesado}

Entre las que se pueden mencionar, son:

a) Porcelana con soporte metálico: Este soporte metálico refuerza mecánicamente la prótesis, y sirve de base para la porcelana en el proceso de cocción.

b) Porcelana sin soporte metálico: Al no poseer soporte pueden hallarse sobre revestimiento, pueden ser fabricadas; ser inyectadas o prensadas (tras un patrón de cera); ser torneadas (por sistemas CAD-CAM); o ser coladas (tiene la misma técnica del colado de metales, con el método de cera perdida). (Andrea, 2012) 
Miguel A. Rodriguez-Llaguno; Jorge L. García-Villamar; Ernesto R. Montece-Seixas; Katherine E. Lima-Tamay

\section{Materiales y métodos}

\section{Porcelanas feldespáticas}

Pueden a su vez clasificarse en: Feldespáticas para fundir sobre metales, por sus bajas propiedades físicas se emplean como revestimiento de un núcleo o base metálica que actúa como infraestructura aportando resistencia.

Tienen un coeficiente de variación térmica similar al metal de base sobre el que se funden para que durante el proceso de cocción se unan íntimamente sin crear defectos que puedan llevar al desprendimiento.

El feldespato es un aluminosilicatos con potasio y/o sodio y en estos casos constituye el componente principal. De la composición de una porcelana de usos generales se conserva, aunque no siempre, el cuarzo, pero como se mencionó es eliminado el caolín. La fusión de los componentes del feldespato (silicio - aluminio - potasio y/o sodio) da origen a un vidrio feldespático (fase o matriz vítrea) y cristales de leucita (fase cristalina).

La fusión del vidrio feldespático con cristales de leucita genera un producto translúcido (recuérdese que la translucidez y el brillo son condiciones básicas para una restauración estética) ya que ambos componentes tienen un índice de refracción de la luz similar. (Hepbrum, 2012)

\section{Cerámicas aluminosas}

- $\quad$ Óxido de aluminio $>50 \%$

- Mayores propiedades mecánicas.

- Aumento significativo de la opacidad.

\section{Cerámicas circoniosas}


- $\quad$ Óxido de zirconio $>95 \%$

- Mecanismo de refuerzo «transformación resistente»

- $\quad$ Alta resistencia a la flexión «acero cerámico»

- $\quad$ Muy opacas.

(Mendez, 2011)

\section{Metal-porcelana}

El metal-porcelana combina la resistencia y exactitud de un colado con la estética de la porcelana. Las restauraciones de metal porcelana están formadas por un colado o cofia, que ajusten en el tallado del diente y por la porcelana adherida por dicha cofia. La cofia metálica se recubre con tres capas de porcelana:

1) Porcelana opaca, que oculta el metal subyacente.

2) Cuerpo o dentina que constituye la menor parte del grueso de la restauración y que es responsable de color y tono.

3) Esmalte incisal, que es una capa translucida de porcelana en la porción incisal del diente.

Las porcelanas deben de tener un punto de fusión bajo y coeficiente de expansión térmica que sea alto, a fin de que sea posible fundirla en las aleaciones adecuadas para cofia.

En la composición de cerámica dental se requiere añadir cantidades mayores de sosa y potasa a fin de aumentar la expansión térmica a un nivel compatible con la cofia de metal. Es importante señalar que las porcelanas de alta expansión tienen una tendencia mayor a desvitrificación por su contenido alcalino; por lo que no se recomienda someterla a cocciones repetidas porque aumenta el riesgo de oscurecer la porcelana, así como cambios en la expansión térmica. 
(Andrea, 2012)

\section{Principios de la preparación}

- $\quad$ Preservar la estructura dentaria

- $\quad$ Retención y estabilidad

- $\quad$ Solidez estructural

- $\quad$ Márgenes perfectos

- $\quad$ Perseverar el periodonto

\section{Surcos guía de profundidad}

Consiste en realizar surcos definidos con una fresa esférica de aproximadamente 1,3-1,5 mm de diámetro previamente conocido. Se recomienda una fresa esférica ya que es la única donde no varía el diámetro al cambiar la angulación. Puede usarse también una cilíndrica solo en sentido recto

Si se requiere un espesor de $1,5 \mathrm{~mm}$, la profundidad de la guía debe ser máximo de $1.3 \mathrm{~mm}$ para en el pulido obtener el espesor ideal. La cantidad de estructura dental removida dependerá del espacio necesario para los materiales de la corona o retenedor

Sector vestibular El surco horizontal enlazará los espacios interproximales corriendo en paralelo al margen gingival y a una distancia aproximada de 1,0 $\mathrm{mm}$ de este. Es importante que este surco siga la dirección de la encía marginal y la convexidad de la superficie. Se realizan 2 o 3 surcos de profundidad paralelos entre ellos y el eje mayor del diente

Sector lingual No se hacen guías paralelas. En un principio se deja el cíngulo sin preparar para evitar la sobredimensión del hombro o chamfer. Luego se procede a la reducción. Si el diente es muy delgado no se hace surco guía horizontal 
Sector incisal Se hace un surco más profundo ya que la reducción va a ser más o menos de $2 \mathrm{~mm}$. Castellani, reduce el tejido intacto delimitado por los surcos guía y se deberá asignar al corte una dirección inclinada de atrás hacia delante (de palatino a vestibular) y de arriba abajo con una fresa troncocónica.

\section{Reducción axial}

Es la fase de extirpación de tejido coronario, dirigiendo el movimiento en paralelo al eje axial del diente. El procedimiento costa de varias fases y comienza con la separación del muñón de los dientes contiguos

Se hace con una fresa troncocónica que se coloca totalmente vertical, para que la conicidad de la preparación sea dada por la fresa. La cual puede ser de tungsteno o de diamante extremo plano o redondeado. Si la fresa es cilíndrica, la conicidad de la preparación dependerá de la angulación que el clínico le dé a la fresa. Continuando, la fresa se introduce con un movimiento suave para que quede un remanente de esmalte, el cual se retirará hasta el final de esta fase. El corte debe seguir la dirección de la papila. Esta reducción continuará hasta llegar a la vertiente lingual siguiendo el margen gingival. La reducción deberá ser suficiente para permitir la fabricación de troqueles y una buena higiene oral. Se debe verificar que el eje del muñón coincida con el eje de los dientes contiguos, para lo cual el operador debe situarse a las 12 del reloj con respecto al paciente

\section{Reducción Vestibular}

Consiste en eliminar los islotes creados por los surcos de profundidad. La fresa se debe tomar en posición oblicua para evitar que toque los surcos de profundidad. Cuando se llega a los surcos se coloca la fresa vertical para realizar el plano cervical e incisal de la preparación. El cervical determina el eje de inserción y paralelismo de la pared lingual, y el incisal permitirá el 
Miguel A. Rodriguez-Llaguno; Jorge L. García-Villamar; Ernesto R. Montece-Seixas; Katherine E. Lima-Tamay

restablecimiento estético y funcional de un diente anterior. Si se dejara recto la prótesis no tendrá uniformidad lo cual permitirá ver la presencia del opacador en las zonas delgadas de la prótesis y pronunciadas de la preparación

\section{Reducción Palatina}

Se presenta en 2 fases

En una primera se define la zona cervical creada o iniciada en la separación del muñón con la fresa paralela el eje del diente, creándose la conicidad ideal con cervical vestibular. Castellani recomienda una terminación en hombro, mientras que Tylman recomienda un "chaflam curvo", también denominado chamfer

En una segunda fase se remueve tejido que va del cíngulo al borde incisal para lo cual se usa una fresa en forma de pelota de rugby de un calibre que reproduzca las dimensiones del pilar. Esta reducción debe proporcionar un espacio interoclusal de $1.0 \mathrm{~mm}$ como mínimo. La cual se verifica únicamente en oclusión y no en movimientos excursivos. Es necesario garantizar el adecuado espacio para el material restaurador elegido, y por consiguiente su durabilidad en boca, disminuyendo posibles fracturas de la cerámica o perforaciones en el metal, o trauma oclusal que afectará también a los dientes antagonistas.

\section{El acabado de la preparación}

Consiste en alisar y suavizar las aristas comprobar el grado de paralelismo y corregir los socavados que pudiera haber. Las aristas dificultan la toma de impresión, el encerado y crea mayor posibilidad de fractura. Castellani recomienda las piedras de Arkansas con abundante refrigeración, también se pueden utilizar fresas troncocónicas de grano fino y de balón de grano fino para la superficie palatina 


\section{Surcos guía de profundidad}

Los de la superficie oclusal deberán tener una profundidad de entre 1,2 y $1,7 \mathrm{~mm}$, ya que, en esta zona, la reducción definitiva ascenderá a 1,5 mm aproximadamente en las cúspides de corte y de 2 mm en las cúspides de estampado. Es importante realizar surcos tanto en las zonas de relieve (cúspides, crestas triangulares), como en las de depresión (fosas, surcos de desarrollo) usando una fresa redonda

\section{Reducción oclusal}

Se lleva a cabo eliminando los islotes de tejido intacto con una fresa troncocónica o en forma de pelota de rugby. Es importante recordar que la superficie reducida debe reproducir en su naturaleza geométrica la morfología oclusal normal se debe revisar el espacio interoclusal en oclusión y en movimientos excursivos para evitar trauma oclusal. (Echeverria, 2014)

\section{Toma de impresión}

Con la utilización de hilos de retracción trenzados (p.ej. Ultrapak, Ultradent Products/USA) 3/0 se consigue una buena reproducción del límite de la preparación. Empleando la técnica de doble hilo se obtienen impresiones con resultados buenos y reproducibles.

Esta técnica consiste en la colocación de un primer hilo delgado en el surco. Permanece colocado ahí durante todo el proceso de toma de impresión. Encima de éste se coloca un segundo hilo más grueso, que se retira unos instantes antes de tomar la impresión. El primer hilo tiene la finalidad de impedir el sangrado del surco.

Al mismo tiempo deberá evitar el retroceso de la encía por encima del límite de la preparación. Para la técnica de la doble mezcla se recomienda utilizar cubetas individuales, elaboradas en el 
Miguel A. Rodriguez-Llaguno; Jorge L. García-Villamar; Ernesto R. Montece-Seixas; Katherine E. Lima-Tamay laboratorio - o como mínimo, individualizar una cubeta de impresión prefabricada (cubeta Rimlock) con un encofrado distal de resina fotopolimerizable o material termoplástico.

La toma de impresión puede realizarse con todos los materiales de impresión utilizados corrientemente para la prótesis fija Con los polisiloxanos puede aplicarse tanto el procedimiento de corrección, como también la técnica de doble mezcla. Con los materiales basados en poliéster únicamente puede utilizarse la técnica de la doble mezcla

Tanto en la técnica correctiva, como también en la técnica de doble mezcla, se recomienda distribuir el material de impresión con un chorro de aire después de aplicar el componente de baja viscosidad. De esta forma, se asegura una buena humectación de la superficie del muñón. (Tellez, 2008)

\section{Resultados}

\section{Impresión primaria}

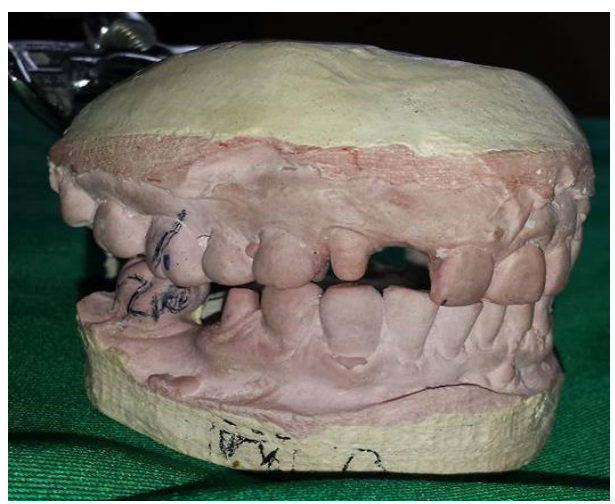

\section{Encerado guía}



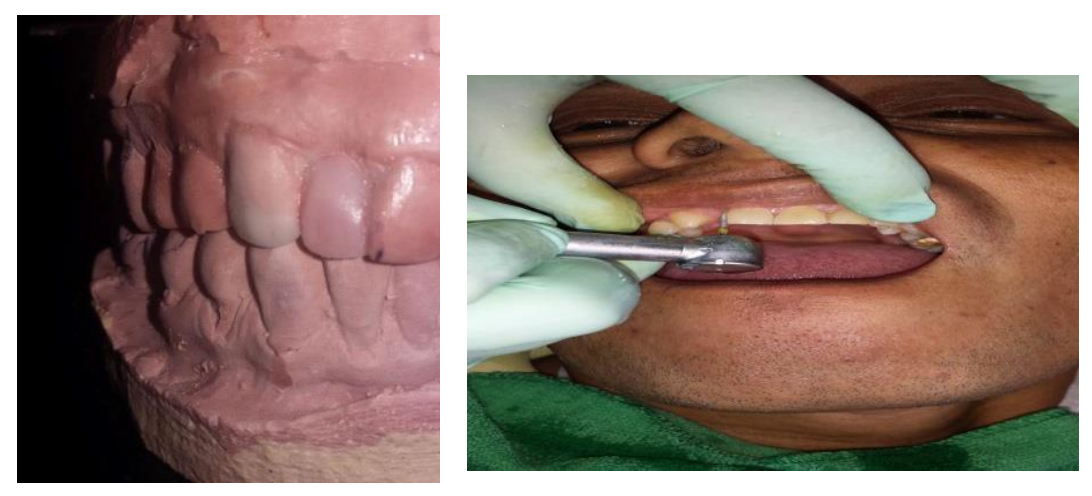

3. Preparación de pieza dentaria con fresa cilíndrica punta redondeada

Preparación de la pieza dentaria lista

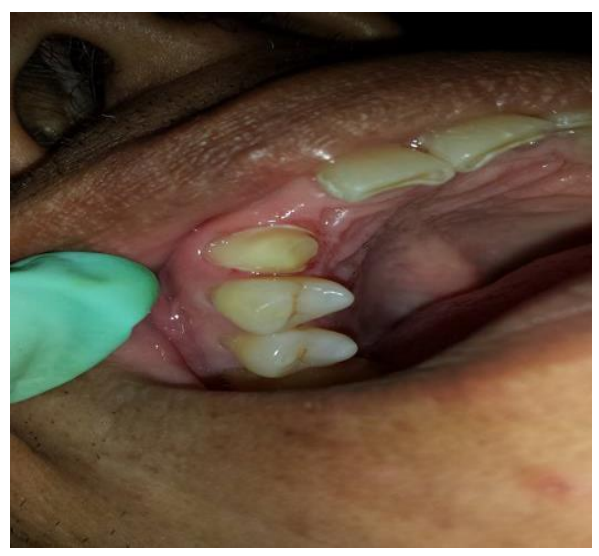

Preparación de la pieza
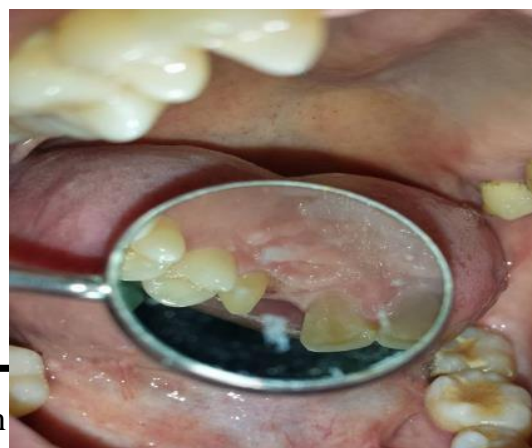

dentaria lista, vista palatina 


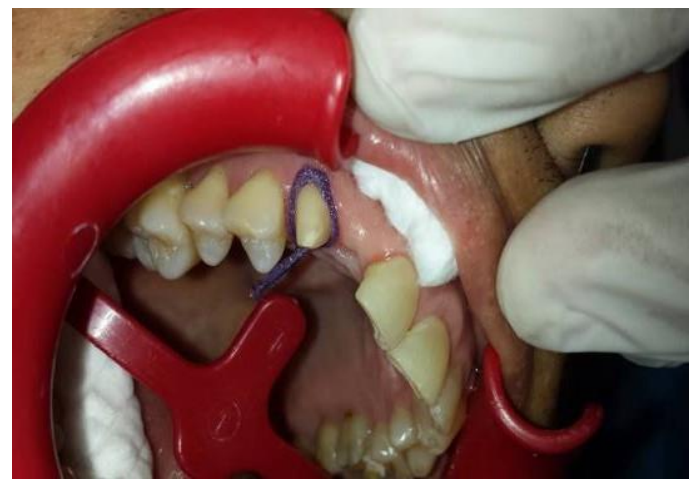

Provisionales

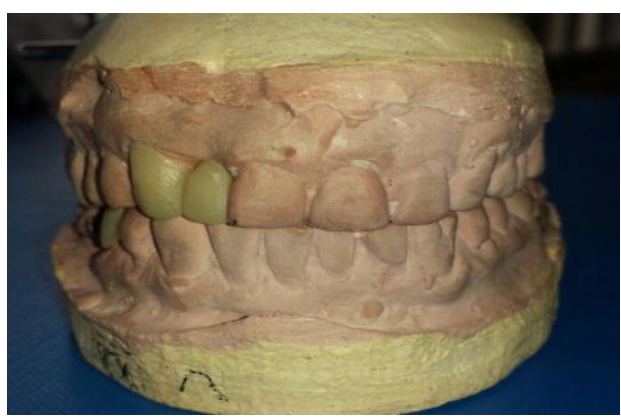

\section{Prueba del metal}

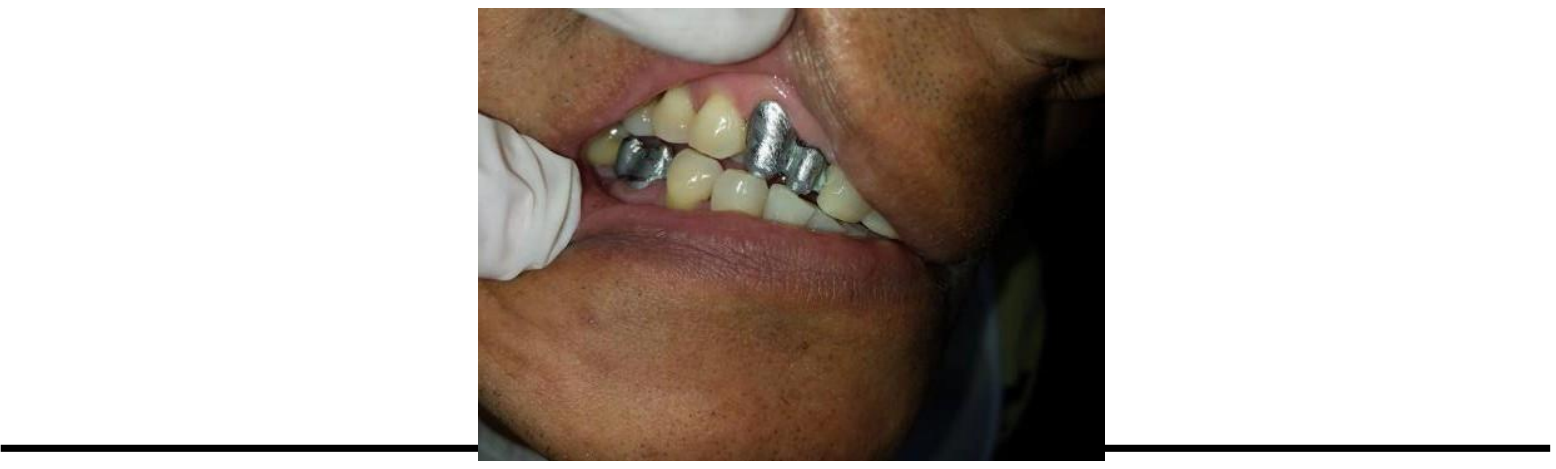

Pol. Con. (Edición núm. 9) Vol. 2, No 7, junio 2017, pp. 111-129, ISSN: 2550 - 682X 


\section{Toma del color}

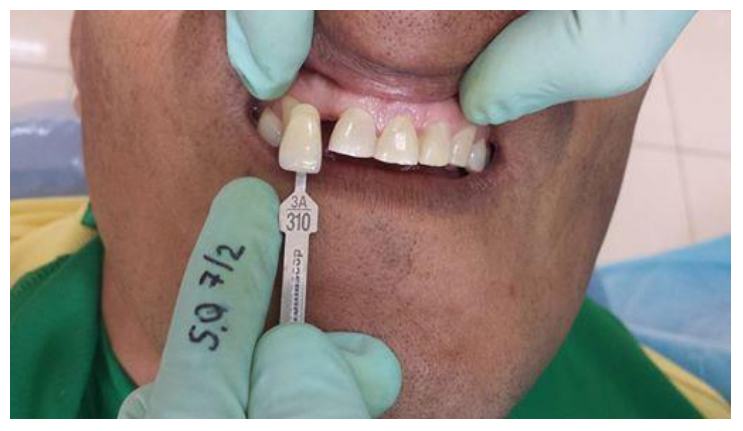

\section{Resultado}

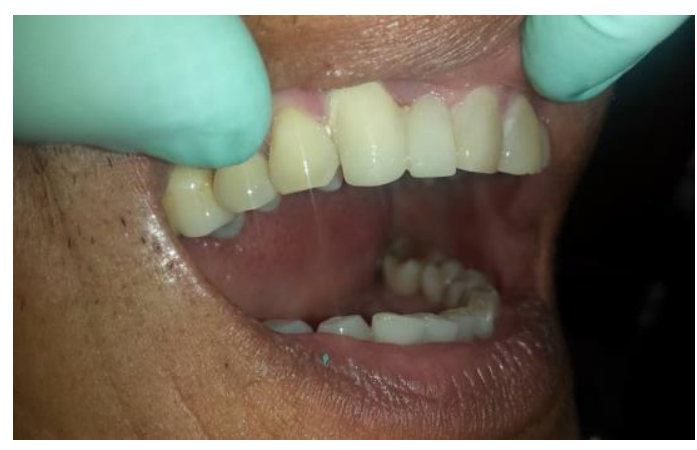

\section{Prueba de oclusión}
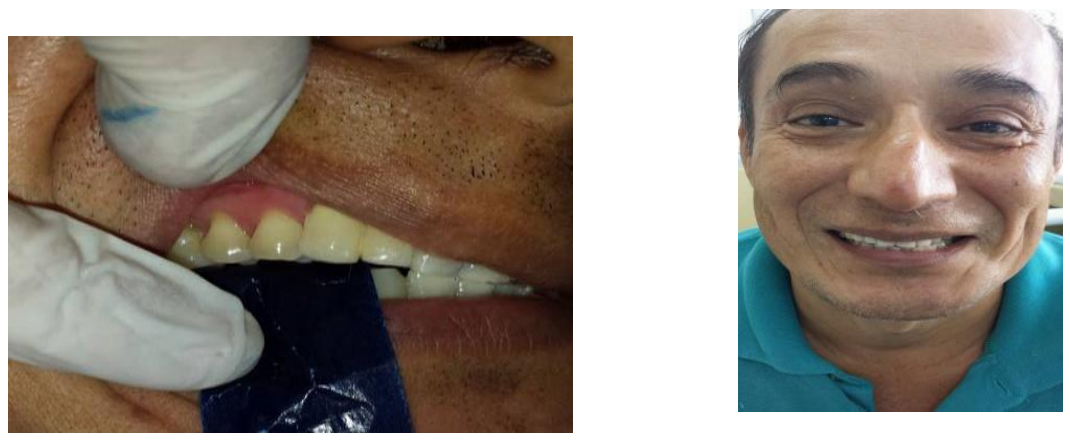


\section{Conclusiones.}

Antes de iniciar cualquier tratamiento es necesario que el paciente se realice una historia clínica, se determine con un examen clínico radiológico o de exploración extraoral y mediante fotografías se documente el caso; ya sea por la restauración parcial o completa dental, no olvidando que los dientes ausentes también cumplían una función que era la de proteger la estructura dentaria remanente. Por otra parte, los orígenes de hábitos bucales pueden ser de diversa índole: emocionales, alérgicos, dentales; por lo que se requiere de una intervención oportuna y de un manejo integral con la intervención de profesionales así Edward Angle estableció una relación basada en la relación de las cúspides entre los primeros molares superiores e inferiores, la cual ha sido tomada como patrón de referencia como la Neutroclusión, Distoclusión, Mesioclusión.

Las porcelanas de tipo feldespáticas no han sido superadas, hasta el día de hoy, en estética por ello son bastante usadas. Existen otras porcelanas como las de Porcelanas aluminosas, Porcelana con soporte metálico, Porcelana sin soporte metálico. Cerámicas aluminosas. Cerámicas circoniosas. Metal -porcelana

\section{Bibliografía}

Andrea, M. (2012). Porcelana en Dientes Anteriores. Boliviana.

Echeverria, D. A. (2014). Restauraion provisionales Temporalizaciòn. issue.

Hepburm, D. A. (2012). Porcelanas Dentales . Ateneo odontologico .

Mendez, D. M. (2011). ceramicas dentales . scielo .

Morán, V. Z. (2013). Tipos de Maloclusiones y hábitos orales más frecuentes, en pacientes infantiles en edades comprendidas entre 6 y 7 años, de la E.B.N Los Salias, ubicada en San Antonio de los Altos, Edo. Miranda, Venezuela . Revista latinoaméricana de ortodoncia y odontopediatria.

Ramirez, G. N.-M. (2012). Protesis Fija ,aparatos ,ortodontico . Laboratorio dental Santa Maria . 
Tellez, L. T. (2008). Rehabilitacion completa de corono metal-ceramica y Protesis parcial Removible .

Villaroel, v. c.-M. (2017). Sistema Ceramico Puro . Venezolana . 